\title{
Alternative Resource Allocation Model for Dynamic Resource-Sharing WSN Systems
}

\author{
Güngör YILDIRIM
}

\author{
Yetkin TATAR
}

\begin{abstract}
One of the major solutions to the interoperability problem of different wireless sensor networks (WSNs) which are in different locations is the use of WSN virtualization techniques. In these types of systems, there is generally a provider system to which the heterogeneous WSNs are registered. Through the provider system, clients can choose different resources existing in the different WSNs and establish their own virtual networks. In virtualization, the resource choice/allocation operation is one of the initial processes. This process is important to clients, the provider and efficient system maintenance. Current resource choice/allocation models used in IoT WSN providers generally take into account client parameters. However, other parameters are also available for optimum resource allocation, including available energy levels of the nodes in the sub-WSNs, data traffic in the sub-WSNs, processing rate of the physical nodes. In addition, the sub-WSNs registered to the provider can have different skills and features. The sub-WSN parameters must be taken into account in the process of the resource choice/allocation. In the paper, an alternative resource allocation model which considers these critical parameters is proposed. In the model, which is analytically explained, both client-side and provider-side parameters are considered. Thus, the systems in the sub WSNs can operate for a longer time and more economically
\end{abstract}

Keywords-WSN, virtualization, IoT, resource sharing

\section{Introduction}

WSN technologies have made a lot of progress for decades, and many software/hardware WSN technologies have been developed in this period. Consequently, big IoT projects such as smart city, smart grids have started using WSNs as basic infrastructure units. This has led to the emergence of immense heterogeneous structures under the IoT roof. As a result, researchers and engineers have had to find solutions to the resource sharing problems of heterogeneous WSN systems. One of the best-known solutions to the problem is WSN virtualization. Virtualization enables different applications to utilize the shared resources available in a system by hiding the infrastructure complexity. It is necessary to evaluate the WSN virtualization methods differently from other well-known virtualization methods of computer technologies.

Güngör YILDIRIM

Firat University, Engineering Faculty, Computer Engineering Dept.

Elazig,Turkey

Yetkin TATAR

Firat University, Engineering Faculty, Computer Engineering Dept.

Elazig,Turkey
The reason for this is that WSNs, by nature, have many restrictions. Especially, critical parameters such as energy and memory limitations, low processing capacity, the large number of nodes must be taken into account at all times. Moreover, this is an optimization problem for WSN virtualization. In general, different types of WSN virtualization methods exist, node-based virtualization (NodBV), network-based virtualization (NetBV) [1-3]. But the methods are not suitable for IoT systems that meet the demands of a large number of clients. For this, middleware based sensor cloud systems are more efficient and effective. Virtualization on cloud systems completely isolates the infrastructure from the clients. Thus, the clients are only interested in resource choice [3-6].

In IoT-WSN provider systems can have different working models. For example, in a sensor cloud system, the periodic information from the nodes is transferred to the interested clients through middleware technologies. In this case, the increase in the number of clients may not affect a lot the working system of the sub-WSNs. In this general working model, a sensor node always acquires information from all the resources regardless of whether they are demanded or not. On the other hand, in some resource-sharing models, the provider or sub-WSNs can dynamically change the working period or node operations. These types of systems run on the principle of both query-answer and periodical data handling (e.g., ZigBee smart systems). In these systems, there is no need to obtain information from all the resources which a node has. Only the demanded resources and their functions are managed. The dynamic working models may be more efficient for interactive IoT WSN provider systems. However, in the dynamic working models, a large number of different clients can lead to unbalanced workloads for sub-WSNs and their resources. The proposed model in the paper considers provider systems that use the dynamic working model. The general working principle of an IoT WSN provider system is shown in Fig. I.

The clients, who want to utilize the system, first pass the steps of registration and resource selection/allocation processes. Today the features of the WSN resources are generally advertised on the web through some internet technologies such as SensorML or other XML/JSON based applications [13]. Clients take advantages of the system by selecting the resources they want. During these processes, the general criteria are client parameters. On the other hand, these types of systems consist of sub-WSNs which are generally heterogeneous. In other words, the sub systems may have different types of features and operations. For example, a sub WSN sharing its own resources can run an owner-defined application. In another example, because of the demand difference, certain parts of a sub WSN can be busier than the other parts of the network. 


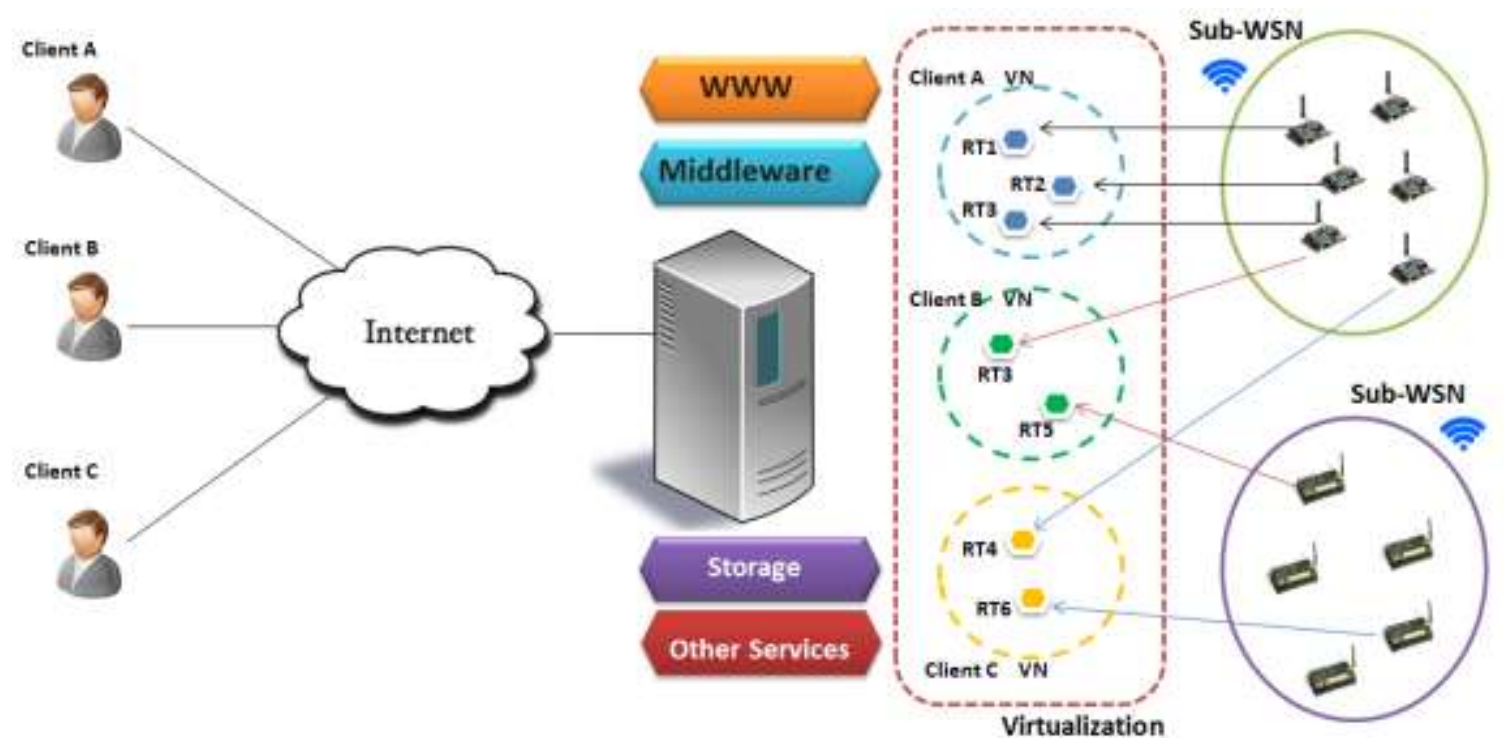

Figure I. The general working principle of an IoT WSN provider system

The situations can affect the general workload and the data traffic of the sub systems. Moreover, sensor nodes may have more than one sensor resources and these resources may be digital or analog. This means that sensor nodes in a sub WSN can have different generated data size. In practice, these examples can be reproduced. Accordingly, the general operation processes of sub-WSNs can be affected adversely by a resource allocation model that does not take the sub WSN parameters into accounts. This means poor and inefficient service quality.

In the study, for the WSN provider systems that use the dynamic working model, an alternative resource selection/allocation model, which considers the system reliability and maintenance, is proposed. In the model two basic criteria are taken into account, the available energy level and working load per node. Some successful analytical approaches to sensor networks systems have been available in the literature $[7,8]$. The proposed model is based on the model in [7]. However, the model considers the general standard working model explained above. Unlike [7], the proposed model takes into account both client parameters and the sub WSN parameters of the provider. For an interactive and effective resource allocation, it is assumed that some conditions are satisfied. First, the provider system has detailed knowledge about the infrastructure of the sub-WSNs, such as transmit power, receiving sensitivity, and coordinate of the nodes. Second, the sub WSNs can have both advanced sensor nodes and poor-skilled nodes.

\section{Analytic Analysis of the Proposed Model}

Each registered resource in the provider side belongs to an owner "Oi", and the subset representing the registered owners is $\mathbf{O}=\{\mathbf{0 1}, \mathbf{O 2}, \ldots \mathbf{O m}\}$. " $\mathbf{m}$ " denotes the number of registered owners. It assumed that " $\mathbf{O}_{\mathbf{i}}$ " has given the necessary permissions about resource sharing to the provider, and at least one owner is registered to the provider. Each resource has a resource type label, $\mathrm{RTi}$. The resource type set is $\mathbf{R T}=\{\mathbf{R T 1}$, RT2,... RTn\}. For example, as $\mathrm{RT}_{\mathbf{1}}$ represents temperature, RT2 can denote humidity. On the other hand, there is a predefined application type subset in the model "ATi", which is defined in RT. The subset is defined as ATi $\in 2^{|\mathbf{R T}|}$. For instance, $\mathrm{AT}_{1}=$ "meteorology", $\mathrm{AT}_{2}=$ "fire", $\mathrm{AT}_{3}=$ "security", $\mathrm{AT}_{4}=$ "general-purpose". When "Oi" is registering to the provider, the shared resources are labeled for the appropriate application type by a system-defined function "gclass(.)". Thus, gclass: $\mathbf{R T} \rightarrow \mathbf{A T i}, \mathbf{A T i} \in \mathbf{2}^{|\mathbf{R T}| \cdot}$ Since ATi $\subseteq \mathbf{R T}, \mathbf{R T i}$ can be a member of more than one application types. This means a temperature sensor can be defined in both meteorology and fire application. In this way, a client can get a resource list including the most appropriate resources according to the application type. Here, the type, "generalpurpose", represents all type of resources, that is, each resource has this label. In addition, in the model a binary variable, "d", is used to denote the current state of the resource, active (1) or passive (0).

Although the clients need the general information of resources, for efficient resource sharing the provider keeps a resource notation, $\mathbf{f r}_{\mathbf{i}}=\left\langle\mathbf{M r}_{\mathbf{i}}, \mathbf{E}_{\mathbf{i}}, \mathbf{L}_{\mathbf{i}}\right\rangle$, which denotes the physical nodes on which the resource is. There, $\mathbf{M r}_{\mathbf{i}}, \mathbf{E}_{\mathbf{i}}, \mathbf{L}_{\mathbf{i}}$ is processing rate (MIPS), the available energy level and the usage cost constant specified by the owner, respectively. Also, another notation that represents the relation between the resource usage and the application type is defined on the system. The notation is $\mathbf{r d}_{\mathbf{i}}=\left\langle\mathbf{g}_{\mathbf{i}}, \mathbf{m}_{\mathbf{i}}, \boldsymbol{\varepsilon}_{\mathbf{i}}\right\rangle$. While gi shows the generated data (bit/s) that the resource can generate data according to application type and running period, mi represents the process rate on the node. $\varepsilon i$ is the resource cost specified by the provider for the application. Before giving the 
resource allocation functions, it is essential to introduce some important parameters to be used.

The most important restriction in resource allocation operations is the available energy levels of the nodes. Under every condition, this needs a monitoring process. The process is accomplished in two ways. The first one is that the sub WSNs inform the provider through given APIs. This is generally possible in the networks which have advanced node technology. The second one is the use of energy level estimation calculations. This method is valid for the networks which include low featured nodes and which do not provide any API that gives the energy level of the node. In this case, three basic states are considered for the energy consumption of a node. Those are the power consumptions for the transmit state $\left(\boldsymbol{P}_{\boldsymbol{i}}^{\boldsymbol{T r}}\right)$, receiving state $\left(\boldsymbol{P}_{\boldsymbol{i}}^{\boldsymbol{R} \boldsymbol{c}}\right)$ and operational state (acquiring and processing). For a sub WSN, $W=\left\{w_{1}, w_{2}, \ldots w_{i}\right.$ , .... $\left.w_{m}\right\}$, the general mathematical model to be used is as follows $[\mathbf{8 , 1 1 , 1 2}]$,

$$
\begin{aligned}
& P_{i}^{T r}=\sum_{h \in W, i \neq h}\left(\beta_{1}+\beta_{2} d_{i h}^{\gamma}\right) P l_{i h} \quad \forall i \in \mathrm{W} \\
& P_{i}^{R c}=\rho \sum_{h \in W, i \neq h} P l_{i h} \quad \forall i \in \mathrm{W} \\
& P_{i}^{t o t}=P_{i}^{T x}+P_{i}^{R x}+f\left(r_{m i}^{i d}\right) \forall i \in W
\end{aligned}
$$

$\boldsymbol{P} \boldsymbol{l}_{\boldsymbol{i h}}$ denotes transmit and receiving data flow in bps. $\boldsymbol{f}\left(\boldsymbol{r}_{\boldsymbol{m i}}^{\boldsymbol{i d}}\right)$, is the function that gives the power consumption during acquiring and processing of a node. The function can be ignored if the resource is a simple sensor. The default values for other constant in the literature are $\boldsymbol{\beta}_{\mathbf{1}}=\mathbf{5 0} \mathbf{~ n j / b i t ~}$, $\boldsymbol{\beta}_{\mathbf{2}}=\mathbf{0 . 0 0 1 3 p J} / \mathrm{bit} / \mathrm{m}^{4}, \quad \rho=50 \mathrm{nj} / \mathrm{bit}$ ve $\gamma=\mathbf{4}[11]$. The important parameter in the equations is $\boldsymbol{d}_{\boldsymbol{i} \boldsymbol{h}}$. In the case where the routing information is known, this value is also known. Otherwise, the value must be estimated according to the worst case. An approximate coverage radius of a node can be calculated if the transmission power $\left(\mathbf{p}_{\mathbf{t x}}\right)$ of the node is known [11-12].

$$
R_{i}^{T}\left(p_{t x}\right)=\left(p_{t x} \cdot \frac{g_{0}}{P_{t r s}}\right)^{1 / \gamma}
$$

There $P_{\text {trs }}$ shows the threshold value for packet receiving on a node (e.g., $-95 \mathrm{dBm}$ ) and $\mathbf{g}_{0}$ is an antenna constant (e.g. 0.0081) [27]. In the case where these parameters are selected as default values, the maximum coverage radius can be calculated. Since the coordinate information of the nodes, $\mathbf{R}_{\mathbf{P o s}}$ $=\left\langle\mathbf{R}_{\mathrm{la}}, \mathbf{R}_{\mathbf{l o n}}\right\rangle$, are registered in the system, the farthest node to which the node can transmit a packet can be found by the Euclidean method. According to this, $\boldsymbol{d}_{\boldsymbol{i n}}$ for the worst case;

$$
d_{i h}^{w r s t}=\arg \max _{h \in W, i \neq h}\left(\left\|\left(R_{p_{\text {os }}}-R_{\text {pos }_{h}}\right)\right\|\right)
$$

In WSN nodes, the average power consumption per MIPS varies according to MCU architectures used and it is generally in the range of $0.1 \mathrm{MIPS} / \mathrm{mW}$ and $2 \mathrm{MIPS} / \mathrm{mW}$ [9].

As mentioned previously, the power consumption of the acquiring and processing operations can be neglected in comparison with $\boldsymbol{P}_{\boldsymbol{i}}^{\boldsymbol{T} \boldsymbol{x}}$ ve $\boldsymbol{P}_{\boldsymbol{i}}^{\boldsymbol{R} \boldsymbol{x}}$ most of the time. Therefore, $\boldsymbol{f}\left(\boldsymbol{r}_{\boldsymbol{m} \boldsymbol{i}}^{\boldsymbol{i d}}\right)$ is disregarded and the estimation total power consumption of a node is $\boldsymbol{P}_{i}^{t o t}=\boldsymbol{P}_{i}^{T x}+\boldsymbol{P}_{i}^{R x}$. On the other hand, this neglect depends on $\mathbf{r d}_{\mathbf{i}} \cdot \mathbf{m}_{\mathbf{i}}$ since some sensor nodes in the sub-WSNs, such as multi-media nodes, can run specific algorithms. Having known the net or estimated energy levels of the nodes, the provider or the owners of the sub-WSNs can want to be listed the resources the energy level of which is over a threshold $(\boldsymbol{\Gamma})$. This is more suitable for the life time of sub-WSNs.

There can be more than one resource on a node, such as a temperature sensor and camera. In this case, a resource on the node may be demanded more than the others. The data generation is closely related to $\left(\mathbf{g}_{\mathbf{i}}\right)$, operation period and application kind. In addition to this, a sub WSN may be running its own specific tasks besides IoT support. Another situation about a node is that the node can have both data acquisition and routing tasks (e.g. ZigBee routers). In a nutshell, the data traffic in the sub-WSNs can dynamically be affected from resource-sharing. In this case, the provider and the clients can agree on a certain traffic rate in order not to hinder the normal sub WSN operations. For this, the flow conservation rule in Eq.6 can be used. According to this, the generated data on a node, $\left(\mathbf{g}_{\mathbf{i}}\right)$, plus the incoming flow rate $\left(\boldsymbol{I}_{\boldsymbol{f}}\right)$ must be equal to the outgoing flow rate [8, 10-12].

$$
\sum_{\substack{h \in W \\ i \neq h}} I_{f}+G \cdot g_{i}=\sum_{\substack{h \in W \\ i \neq h}} O_{f}
$$

A sub-WSN owner and the provider can agree on a maximum constant value " $\boldsymbol{G}_{\boldsymbol{m a x}}$ " . Thus, it is expected that $\mathbf{G}$ is lower than $\boldsymbol{G}_{\boldsymbol{m a x}}$ at all time $\left(\mathbf{G} \leq \boldsymbol{G}_{\max }\right)$. This is an optional restriction and specifies at what level a sub WSN can integrate with IoT systems. After the basic and optional parameters have been specified, the resources on the system are denoted by a 6 - tuple;

$$
r=\left\langle i d, o, t, d, f r, R_{p o s}>\mathrm{t} \in \mathrm{AT}, \mathrm{o} \in \mathrm{O}, \mathrm{r} \in R_{T}\right.
$$

There, " $\mathrm{t}$ " is a tuple showing the application set of which the resource is a member. A resource is the member of at least one AT. On the other hand, the application parameters show the general feature of the virtual network and it is denoted as follows;

$$
U=<U_{\text {id }}, U_{\text {type }}, U_{\text {area }}, r d>
$$

Each client application has a unique "id". When a user application is registered, the system assigns appropriate "rd" 
parameters for the application. $U_{\text {area }}$ is the valid area in which the client is interested. The parameter is denoted by $4-$ tuple, $\boldsymbol{U}_{\text {area }}=<\operatorname{Cor} 1, \operatorname{Cor} 2, \operatorname{Cor} 3, \operatorname{Cor} 4>$. Besides, $\boldsymbol{U}_{\text {type }}$ represents the application type the user has selected, $U_{\text {type }}=A T_{i} \in 2^{|R T|}$.

The resource allocation function is shown by $\boldsymbol{f}_{\boldsymbol{S}}($.$) , and$ involves a sequence of three intermediate functions. The intermediate functions are $\boldsymbol{f}_{\mathbf{1}}(),. \boldsymbol{f}_{\mathbf{2}}(),. \boldsymbol{f}_{\mathbf{3}}($.$) Here,$ $\boldsymbol{f}_{\mathbf{1}}($.$) creates the set of the resources corresponding to the$ application type, $\boldsymbol{f}_{\mathbf{1}}():. \mathbf{U} \rightarrow \mathbf{R}_{\mathrm{T}}{ }^{\prime}, \mathbf{R}_{\mathrm{T}}{ }^{\prime} \subset \mathbf{R}_{\mathrm{T}}$.

$$
f_{1}\left(U . U_{\text {type }}\right)=\left\{r \mid \boldsymbol{r} . t=U_{\text {type }}, \quad r \in R_{T}\right\}=\mathrm{R}_{\mathrm{T}}
$$

Thus, each selected resource supporting the application kind is the member of $\mathbf{R}_{\mathbf{T}}{ }^{\prime}$. Then, the energy and data generation levels of the nodes to which the resources belong are controlled by $f_{2}($.$) . As a result of the function operation,$ $\mathrm{R}_{\mathrm{T}}{ }^{\prime \prime}$ is obtained. Each resource in $\mathrm{R}_{\mathrm{T}}{ }^{\prime \prime}$ has values over the entire predefined threshold constant. The definition of the function is $\boldsymbol{f}_{\mathbf{2}}$ (.) : $\mathbf{R}_{\mathbf{T}}{ }^{\prime}, \rightarrow \mathbf{R}_{\mathbf{T}}{ }^{\prime \prime} \in \mathbf{2}^{\left|R T^{\prime \prime}\right|}$. Thus,

$$
\begin{gathered}
f_{2}\left(f_{1}\left(U . U_{\text {type }}\right)\right)=\left\{r \mid r \in \mathrm{R}_{\mathrm{T}}^{\prime}, \mathrm{R}_{\mathrm{T}}^{\prime} \subset\right. \\
R_{T}, g\left(r, r d_{i} \cdot g_{i}\right) \leq G_{\max } \\
\left.M\left(r . f r . E_{i}\right) \leq \boldsymbol{\Gamma}\right\}=\mathrm{R}_{\mathrm{T}}^{\prime \prime}
\end{gathered}
$$

$\mathbf{g}($.$) gives the updated "G" value according to the data$ generation and the interested resource parameters. $\mathbf{M}($.$) is the$ function that gives the current energy level of the node to which the resource belongs. The value is obtained through either owner APIs or the estimation calculations as explained above. The functionality of $\boldsymbol{f}_{\mathbf{3}}($.$) is to control the location$ and the current state of the resources. According to this,

$$
\begin{aligned}
f_{S}(U)= & f_{3}\left(f_{2}\left(f_{1}\left(U . U_{\text {type }}\right)\right)\right) \\
= & f_{3}\left(f_{2}\left(\mathrm{R}_{\mathrm{T}}^{\prime}\right)\right) \\
= & f_{3}\left(\mathrm{R}_{\mathrm{T}}^{\prime \prime}\right) \\
= & \left\{r \mid \boldsymbol{r} \in \mathrm{R}_{\mathrm{T}}^{\prime \prime}, \mathrm{R}_{\mathrm{T}}^{\prime \prime} \subset R_{T}^{\prime} \subset R_{T}, \text { r.Pos } \subset\right. \\
& \left.\quad U . U_{\text {area }} \text { r.d }=1\right\}=\mathrm{R}_{\mathrm{T}}^{\prime \prime \prime}
\end{aligned}
$$

As a result, the resource allocation process, which considers the sub-WSNs and the client application parameters, is completed. In the WSN providers with the dynamic working model, if the standard models are used, the input is $\overline{\boldsymbol{U}}=<$ $\overline{\boldsymbol{U}_{\boldsymbol{l d}}}, \overline{\boldsymbol{U}_{\text {type }}}, \overline{\boldsymbol{U}_{\text {area }}}>$, and the result is $\boldsymbol{f}_{\boldsymbol{S}}(\overline{\boldsymbol{U}})=\overline{\boldsymbol{R}_{\boldsymbol{T}}}$. In the case of considering the parameters of $\boldsymbol{\Gamma}$ and $\boldsymbol{G}_{\text {max }}$, however, the result is $\boldsymbol{f}_{\boldsymbol{S}}(\boldsymbol{U})=\mathbf{R}_{\mathrm{T}}^{\prime \prime \prime}$ and $\left\{\boldsymbol{r} \mid \boldsymbol{r} \in \mathbf{R}_{\mathbf{T}}^{\prime \prime \prime}, \boldsymbol{g}\left(\boldsymbol{r}, \boldsymbol{r} \boldsymbol{d}_{\boldsymbol{i}} \cdot \boldsymbol{g}_{\boldsymbol{i}}\right) \leq\right.$ $\left.\boldsymbol{G}_{\text {max }} \wedge \boldsymbol{M}\left(\boldsymbol{r} . \boldsymbol{f r} . \boldsymbol{E}_{\boldsymbol{i}}\right) \leq \boldsymbol{\Gamma}\right\}$. In other words, since the nodes that do not satisfy the conditions are left out of assessment, the definition of the result set is $\mathbf{R}_{\mathbf{T}}^{\prime \prime} \subseteq \overline{\boldsymbol{R}_{T}}$.

\section{Conclusion}

The WSN provider systems, which provide resource sharing through virtualization methods, generally consist of sub-WSNs having different technologies. The resource allocation models used in these types of systems generally consider clients parameters. On the other hand, along with the resource sharing, the sub WSNs can have their own application goal or different network traffic character. Moreover, a sensor node can carry different types of sensor resources, which this may lead to different data size, operation load, and energy consumption. The energy levels of the nodes, the most important parameter for a WSN, have to be monitored by the provider system. If this is not possible, then energy estimation calculations must be used. This is essential to provide the system reliability and maintenance.

IoT-WSN provider systems can have different working models. For example, in a sensor cloud system, the periodic information from the nodes is transferred to the interested clients through middleware technologies. In this case, the increase in the number of clients may not affect a lot the working system of the sub-WSNs. In this general working model, a sensor node always acquires information from all resources that it has, regardless of whether the resources are demanded or not. On the other hand, in some resource-sharing models, the provider or sub-WSNs can dynamically change the working period or node operations. These types of systems run on the principle of both query-answer and periodical data handling (e.g. ZigBee smart systems). In these systems, there is no need to obtain information from all the resources which a node has. Only the demanded resources and their functions are managed. These dynamic working models may be more efficient for interactive IoT WSN provider systems. However, in the dynamic working models, a large number of different clients can lead to unbalanced workloads for sub-WSNs and their resources. The proposed model in the paper considers the provider systems that use the dynamic working model.

In the paper, for WSN provider systems using the dynamic working model, an alternative resource selection/allocation model, which take into account important parameters such as energy level consumption, data traffic in the network, have been proposed. The model is an improved version of the resource allocation model proposed in [7]. The model provides an efficient resource allocation for a better fault tolerance and infrastructure maintenance. The model can be used in both the simulations and applications of the WSN providers that include different types of resources.

\section{Acknowledgment}

This study has been supported by the project of dept. of FUBAP of F.U, "Mobile Communication Technologies and Wireless Sensor Networks Laboratory”, project no: MF1420. 


\section{References}

[1] Khan I., Belqasmi F., Glitho R., Crespi N., Morrow M. and Polakos P., "Wireless Sensor Network Virtualization: A Survey", IEEE Communications Surveys \& Tutorials, doi: 10.1109/COMST.2015.2412971, 2015

[2] Khalid Z., Fisal N. and Rozaini M.,"A Survey of Middleware for Sensor and Network Virtualization". Sensors, doi:10.3390/s141224046, 2014.

[3] I Islam M. M., Hassan M.M., Lee G.W. and Huh E.N., “A Survey on Virtualization of Wireless Sensor Networks", Sensors, doi:10.3390/s120202175, 2012.

[4] Evensen, P. and Meling, H., "Sensewrap: A service oriented middleware with sensor virtualization and self-configuration", 5th International Conference on Intelligent Sensors, Sensor Networks and Information Processing (ISSNIP), 261-266, 2009

[5] Sarakis L. and Zahariadis T., "VITRO architecture: Bringing virtualization to WSN world", doi:10.1109/MASS.2011.96 , 2011

[6] Leontiadis I., Efstratiou C., Mascolo C. and Crowcroft J., "SenShare: Transforming Sensor Networks into Multi-application Sensing Infrastructures". Wireless Sensor Networks, doi: 10.1007/978-3-642-281693_5, 2012

[7] S. Misra, , Subarna Chatterjee and M.S. Obaidat," On Theoretical Modeling of Sensor Cloud: A Paradigm Shift From Wireless Sensor Network", IEEE Systems Journal, 2014, DOI: 10.1109/JSYST.2014.2362617, pp:1-10,

[8] C. Delgado , J.R.Gállego, M.C. a , J. O. , S. B. and M.Cesana, "On optimal resource allocation in virtual sensor networks", Ad Hoc Networks, 2016, pp: 23-40

[9] L. Binternagel, "Energy efficient sensor nodes", Proceedings SENSOR 2009 Volume 2 - C4.2, pp: 163-165

[10] Y.S. Yun and Ye Xia, "Maximizing the Lifetime of Wireless Sensor Networks with Mobile Sink in Delay-Tolerant Applications", IEEE Transactions on Mobile Computing, Vol.: 9, I.: 9, 2010

[11] Y. Shi , T. Hou, J. Liu, S. Kompella, Bridging the gap between protocol and phys- ical models for wireless networks, IEEE Trans. Mobile Comput. 12 (7), 2013, pp: 1404-1416

[12] T. Hou , Y. Shi , H. Sherali, Rate allocation and network lifetime problems for wireless sensor networks, IEEE/ACM Trans. Netw. 16, 2008, 321-334.

[13] http://www.opengeospatial.org/standards/sensorml 